\title{
Research on Desktop Based Virtual Maintenance Training System for Military Aircraft
}

\author{
Dunming Tan ${ }^{1, *}$, Yonghong Zhou ${ }^{2}$ and Xiaoju Yong ${ }^{1}$ \\ ${ }^{1}$ Aviation Maintenance NCO Academy, Air Force Engineering University, Henan Xinyang, China \\ ${ }^{2}$ PetroChina SiChuan Petrochemical Company, Sichuan Chendou, China \\ ${ }^{*}$ Corresponding author
}

\begin{abstract}
Desktop based virtual maintenance training system for military aircraft system was studied to make the developing easier. First, the virtual maintenance scene was simplified and rendered in real time. Second, the mechanical assemble relationship and sequence was modeled by petri network. Third, different training modes and analyze methods were designed for evaluation of the training effects. The result show by this desktop based system, the development can be easier for maintenance trainers who have not got professional virtual reality skills; in addition this method has a strong universality.
\end{abstract}

Keywords_virtual reality; maintenance; military aircraft; desktop based

\section{INTRODUCTION}

The development of virtual maintenance training system is difficult for maintenance trainers who haven't got professional virtual reality (VR) skills and experiences. This paper is aimed to establish a standard way to develop desktop based virtual maintenance training system of military aircraft for maintenance trainers. The research was mainly focused to make the development easy and universal for maintenance trainers to make the virtual maintenance training system more useful and accurate.

\section{RELATED WORK}

The first challenge for virtual maintenance training systems is the complex scene, which is difficult for real-time rendering, especially for aircraft maintenance, which has millions of mechanical models [1]. There are mainly two ways to solve this problem. The first way use model partition, voxel based GPU ray-tracing on high performance computers [2-3], which achieves preferable effects, however the experiment equipment is rare, and these technics are not adopted in most commonly used virtual reality software. The second way use traditional model simplification, level of detail and visibility culling [4-5], these are easy to integrated to virtual reality software or applications, and is more suitable for virtual maintenance training systems.

Virtual reality system including immersive and the desktop based. The former gives the trainer a vivid experience, but it needs virtual reality devices such as motion tracking, cave or tiled-display system, which make the total costs of virtual maintenance training system too high to afford [6]. So for virtual maintenance applications, the desktop based system is enough for most circumstances [7-8].

The key issue for virtual maintenance training system is how to simulate the maintenance activity by computer. Especially install and remove of mechanical parts, weather a operated part can be installed or not, do it has mechanical constrains by other parts? Petri network based assemble relationship was adopted by most researches to solve this problem [9]. For maintenance simulation, the tools and devices used should also be take into consideration while building the assemble relationship [10]. For desktop based virtual reality systems, because there is no motion tracking device, all the interaction between the trainer and the virtual scene is accomplished by keyboard and mouse [11].

\section{Virtual Maintenance Scene}

\section{A. Building the Virtual Maintenance Scene}

The first step for virtual maintenance training system is to build the virtual scene, however for aircraft maintenance applications, the virtual scene is usually very large. Through there are researches on massive aircraft models [2-3], it is not the focus of virtual maintenance training systems. Simple and efficient means are more suitable. The aircraft is consists of several structure systems such as the fuselage, the landing gear, the hydraulic system, the fuel system and so on. Each system is almost independent of others, and the maintenance work is usually carried out on parts of a specific system. So we can classify the maintenance work according to its aircraft system, each maintenance task only loading its related system, not the whole aircraft structure. This can greatly reduce the complexity of virtual scene, so the maintenance scene can run in real-time on most personal computers. (See Figure I) 


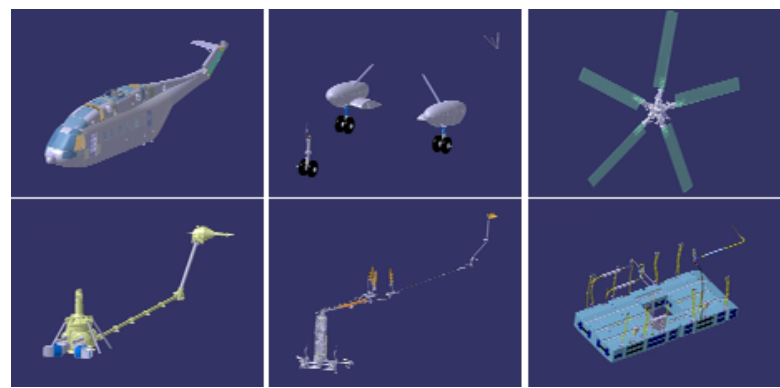

FIGURE I. MAINTENANCE SCENE OF RELATED SYSTEM ACCORDING TO THE TASK

However, some maintenance work is accomplished on several systems, if the scenes of related systems were all loaded at one time, there were massive geometries and cannot be rendered in real-time. Under these circumstances, the stage of computer games is a good solution to learn from. The maintenance is consists of several steps and each step only interacts with one specific system scene. So the next maintenance scene can be loaded in when the previous maintenance step was finished and unloaded.

\section{B. Realistic Rendering of the Virtual Maintenance Scene}

The aircraft models were usually built with CAD software, and are far from realistic. The materials of geometries need modification for realistic, especially the ambient, diffuse and specular reflection parameters. The skin of aircraft fuselage has paintings, which were impossible to model in CAD software. Besides, there are many detailed parts such as the screws, rivets, if all with real models then the scene will be too complex to render. These two issues can be solved by texture mapping in computer graphics without any additional modeling to the geometry part, and make the whole aircraft vivid. (See Figure II)

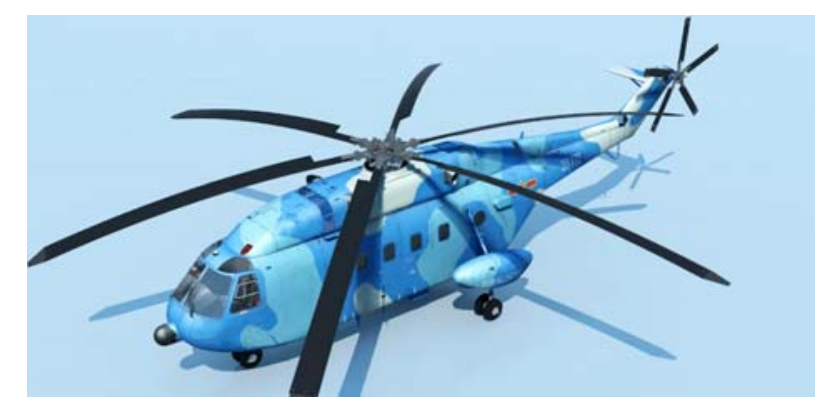

FIGURE II. REALISTIC RENDERING WITH TEXTURE MAPPING FOR PAINTINGS

For mechanical fault such as the break, flaws and the holes, it can be modeled by Boolean operations in the modeling software. Before the maintenance, a prebuilt fault model was used for rendering, after the maintenance another original model will replace the fault model. This can also be applied to mechanical processing such as drilling, riveting, cutting and so on.

\section{Virtual Maintenance Simulation}

\section{A. Modeling the Assembly Constrain Relationship}

For aircraft virtual maintenance applications, it is difficult to simulate the mechanical assembly constrain relationship as on the real maintain target. For example, a bolt cannot be uninstalled unless the nut was demounted. On the real structure the restrain is physically exists, the maintainers just operate on it, however for virtual maintenance applications, the assembly constrain relationship should be built first with computer for further simulation to avoid illegal assembly sequence and operations. For mechanical assembly, especially for maintenance, the install and uninstall has fixed or designed sequence, namely a direct graph or network. This is quite suitable for petri network to build the assembly constrain relationship [9]. A Petri network consists of three typical key elements, namely the order executed nodes, the parallel executed nodes and the conflict executed nodes, as shown in Figure III.

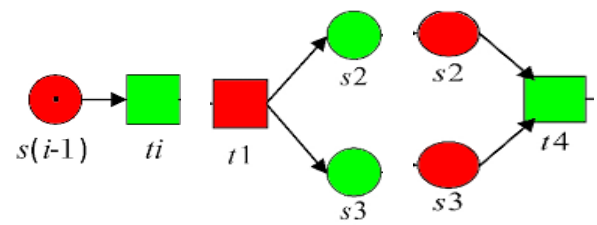

FIGURE III. THREE TYPICAL ELEMENTS OF PETRI NETWORK

Take installation for example, the order executed nodes for assemble constrain relationship means a part must be installed then another part can be installed, the two parts were installed in order. The parallel executed nodes for assemble constrain relationship means two parts can be installed in either sequence, there is no influence or constrain to each other. The conflict executed nodes for assemble constrain relationship means only several parts were all installed, and then can the next parts be installed. In real maintenance applications, the assembly relationship is a combination of the three typical elements of petri networks. By the petri network modeling of assembly constrain relationship, the maintenance activity can be simulated. A maintained part can be installed or uninstalled by first querying weather the assembly constrains were all satisfied from the petri network. The information of assembly constrain relationship can be stored as xml and load dynamically during simulation.

\section{B. Maintenance Interaction}

Another key issue for virtual maintenance application is maintenance interaction. For example, how to simulate maintenance operations with tools on computer? Because the virtual maintenance application have to take the development and redistribution cost into consideration, the desktop based virtual maintenance training system is preferred, which do not use expansive VR interaction devices. Therefore, the mouse and the keyboard can only be used. The maintenance activities were triggered by mouse clicking on the operated part. A maintenance step usually uses specified tools, so the virtual maintenance interaction must choose the right tools first, and 
then the mouse clicking on the desired part can be maintained, shown in Figure IV.

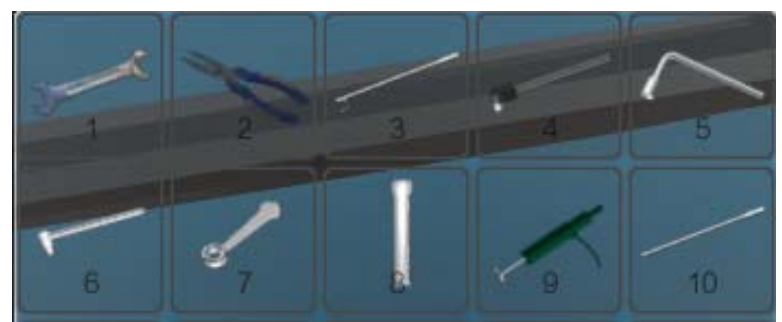

FIGURE IV. MAINTAIN STEP BY FIRST CHOOSING THE CORRECT TOOLS

When the maintenance interaction activity was triggered, the operated parts should respond to its corresponding install or uninstall movement. For example, a nut should rotate and move from a bolt while install, and a bolt should only move and leave the fixed part while uninstall. Then how to simulate the movement of mechanical parts during maintenance? Usually predefine animations set in modeling software such as 3d Max is used to animate the maintain behavior, however, this requires professional 3d model building and animation technicians. In order to make this work simple enough for the maintenance trainers who have not got professional virtual reality or 3d modeling skills, predefined movements were defined in a xml file, which contains the movement type, movement parameters, so the maintenance trainers just need to concentrate on the maintenance work not the VR development skills.

\section{ViRTUAL MAINTENANCE TRAINING}

\section{A. Maintenance Training Modes Design}

The aircraft maintenance is complicated and strict so that the learner cannot achieve the required ability without first learning, then training and finally examine. Therefore, the virtual maintenance training system should also contain corresponding function. The learning mode mainly teaches the learner the function, structure and principle of the maintaining aircraft parts and systems. The training mode gives the learner a hint of the next operated parts and tools used with text information and flicking of the parts in 3D scene, shown in Figure V. The examine mode simulates as the real maintaining work with no hint at all, just text of next procedure.

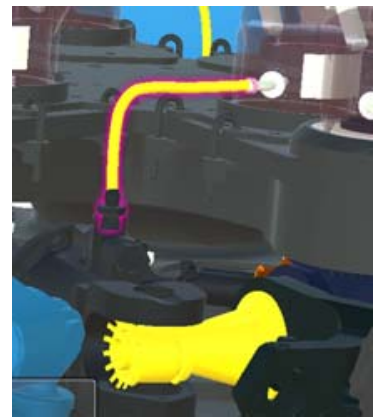

FIGURE V. TRAINING MODE WITH A HINT OF NEXT OPERATED PARTS

\section{B. Assessment of the Maintenance Training}

The aircraft virtual maintenance application should not only be used for training, the training effect and result should also be analyzed for further improvement in teaching and reinforce in training. Thus, the training result needs assessment.

For the desktop based virtual maintenance applications, there are three key elements for assessment, namely the maintaining operation errors, maintaining time spent and the maintaining scores. By these assessments, the maintenance trainer can take additional teaching and training to the maintenances with low scores and long time.

\section{EXPERIMENTS}

Based on the research work, a military helicopter virtual maintenance application was developed. The prototype application was consists maintenance of the whole mechanical system of the helicopter, namely the fuselage, the landing gear, the rotor, the transmission system and so on. Each system has several typical maintenance works, which simulates the whole maintain work on computer by mouse clicking and dragging.

The maintain simulation use only $\mathrm{xml}$ files for configuration, which is simply for maintenance trainer to develop. And the virtual maintenance training system can be run on personal computers without any VR devices; therefore it can be distributed on many computers for large scale training, especially for military aircraft maintenance training, which has not real aircraft to work with and can only be solved by virtual maintenance with limit costs and large amount of learners.

\section{CONCLUSION AND FUTURE WORK}

This paper studies the key technologies to develop desktop based virtual maintenance training systems of military aircraft. It proves that an affordable virtual maintenance with low develop difficulty is possible.

Future work consists of maintenance simulation of the user for visibility and accessibility analyzes and constrain, which can be achieved by a virtual maintaining human. What's more, a VR motion tracking device is also needed if the costs is not a problem.

\section{REFERENCES}

[1] Gobbetti, E. and Marton, F. Far Voxels - A Multiresolution Framework for Interactive Rendering of Huge Complex 3d Models on Commodity Graphics Platforms [J]. ACM Transactions on Graphics, 2005, 24(3):878-885

[2] Fenglin Tian, Wei Hua, Zilong Dong, et al. Adaptive voxels: interactive rendering of massive 3D models [J]. The Visual Computer, 2010, 25(6): 409-419

[3] Wald, I. and Dietrich, A. and Slusallek, P. An Interactive Out-of-Core Rendering Framework for Visualizing Massively Complex Models [C]. Proceedings of the Eurographics Symposium on Rendering, 2004

[4] C. Peng, and Y. Cao. GPU-based streaming for parallel level of detail on massive model rendering [C]. Eurographics 2012, the 33rd Annual Conference of the European Association for Computer Graphics, 2012

[5] Tan Dunming, Zhao Gang. Real-time Rendering of Massive Aircraft Model for Assembly [J]. Journal of Computer-Aided Design \& Computer Graphics, 2012, 24(5): 590-597 
[6] Muhanna A. Muhanna. Virtua reality and the CAVE: Taxonomy, interaction challenges and research directions [J]. Journal of King Saud University, Computer and Information Sciences (2015) 27:344-361

[7] J.R. Li, L.P. Khoo, S.B. Tor. Desktop virtual reality for maintenance training: an object oriented prototype system (V-REALISM) [J]. Computers in Industry, 2003, 52: 109-125

[8] Peng Gaoliang, Wang Gongdong, Liu Wenjian, Yu Haiquan. A desktop virtual reality-based interactive modular fixture configuration design system [J]. Computer-AidedDesign, 2010, 42:432-444

[9] Lu Zhong , Sun Youchao, Okafor Ekene Gabriel, Wu Haiqiao. Disassembly sequence planning for maintenance based on metaheuristic method [J]. Aircraft Engineer ing and Aerospace Technology, 2011, 83(3): $138-145$

[10] LIU Bei-bei,TIAN Ling,YANG Yu-hang. Key technologies for aviation virtual maintenance training system [J]. Computer Integrated Manufacturing Systems, 2012, 01:47-57

[11] ZHAO Hong-fei, ZHANG Qi, WANG Hai-tao, FANG Bao-shan. Desktop virtual maintenance training system for construction machinery [J]. Chinese Journal of Construction Machinery, 2013, 11(5): 457-462 